\title{
MAPK immunoreactivity in streptozotocin-induced diabetic rat testis ${ }^{1}$
}

\author{
Yelız Bozdemır Donmez ${ }^{I}$, Gulnur Kizilay ${ }^{\mathrm{II}}$, Yeter Topcu-Tarladacalisir ${ }^{\mathrm{III}}$
}

DOI: http://dx.doi.org/10.1590/S0102-8650201400160004

'Fellow PhD degree, Department of Histology and Embryology, Faculty of Medicine, Namık Kemal University, Tekirdag-Turkey. Intellectual and scientific content of the study, technical procedures, acquisition and interpretation of data, manuscript writing.

${ }^{I I} \mathrm{PhD}$, Associate Professor, Department of Histology and Embryology, Faculty of Medicine, Trakya University, Edirne-Turkey. Intellectual and scientific content of the study, design the protocol, technical procedures, acquisition and interpretation of data, manuscript writing, critical revision, final approval of the version to be published.

IIIPhD, Assistant Professor, Department of Histology and Embryology, Faculty of Medicine, Trakya University, Edirne-Turkey. Interpretation of data, manuscript writing, critical revision.

\section{ABSTRACT}

PURPOSE: To evaluate the alterations of two mitogen-activated protein kinases (MAPK)s, extracellular signal regulated kinase (ERK) and c-Jun NH2 terminal kinase (JNK), in the testes of male rats with experimental diabetes.

METHODS: Twenty males Sprague-Dawley rats were randomly divided into a control group $(\mathrm{n}=8)$ and a diabetes group (administration of $40 \mathrm{mg} / \mathrm{kg}$ /day streptozotocin (STZ) for five sequential days, $\mathrm{n}=12$ ). After six weeks, testicular biopsy samples were obtained for light microscopy and immunohistochemical methods.

RESULTS: The PCNA (proliferating cell nuclear antigen) index was significantly decreased in the diabetes group ( $\mathrm{p}=0.004)$ when compared to the control group. Both total ( $t$ )-ERK and phosphor (p)-ERK immunoreactivities were significantly decreased in the diabetes group $(p=0.004, p<0.001$, respectively). The t-JNK immunoreactivity was unchanged in both groups ( $p=0.125)$, while $p-J N K$ immunoreactivity was significantly increased in the diabetic group $(\mathrm{p}=0.002)$.

CONCLUSIONS: The decrease of androgen levels in the course of diabetes may contribute to the decrease of the immunoreactivities of t-ERK and p-ERK. JNK may be activated due to the changes in various cytokines and chemochines that participate in the oxidative stress process of diabetes. Therefore, testicular apoptosis may occur and lead to infertility associated with diabetes.

Key words: Diabetes mellitus. Mitogen-Activated Protein Kinases. Extracellular Signal-Regulated Kinases. JNK Mitogen-Activated Protein Kinases. Streptozocin. Testes. Rats. 


\section{Introduction}

Diabetes mellitus (DM) represents one of the greatest threats to health worldwide. The growing incidence of DM will inevitably result in an increased prevalence in men of reproductive age ${ }^{1}$. Male infertility is a common complication of diabetes. The most frequent effects ofDM on the male reproductive system include decreased levels of testosterone, lower testicular weight, abnormal spermatogenesis, alterations in sperm count and motility, and histopathological changes in testis tissue components ${ }^{2-5}$. Abnormalities in spermatogenesis due to increased apoptosis have been demonstrated in certain studies, s, $3,6-10^{\text {. }}$ Testicular apoptosis may be the major component of the infertility. Infertilityin young men with type 1 or type 2 diabetes is showing a dramatic increase in recent years.

The nuclear matrix protein known as proliferating cell nuclear antigen (PCNA) is an indicator for DNA synthesis with its maximal release in the $\mathrm{S}$ and $\mathrm{G} 2$ phases of the cellular cycle; it cannot be detected in the G0 phase. PCNA immunoreactivity should be assessed to understand the effects of DM on spermatogenesis and proliferation ${ }^{11}$.

Mitogen-activated protein kinases (MAPK's) regulate various physiological and pathological cellular actions with their three subgroups; extracellular signal regulatory kinase (ERK), c-Jun NH2 terminal kinase (JNK)/Stress Activated Protein Kinase (SAPK), and p38 MAPK. ERK supports cellular proliferation by responding to epidermal growth factors (EGFs) and other extrinsic mitogenic signals. JNK and p38 MAPK are identified as "stress activated kinases" that are generally related to inflammation and apoptosis ${ }^{12}$. They participate in the apoptotic signal transduction as a response to various factors, including inflammatory cytokines, ultraviolet lights, radiation, heat, hydrogen peroxide, and osmotic shock ${ }^{13}$.

This study compared the immunoreactivities of ERK and JNK to evaluate the effects of DM on male germinal cells.

\section{Methods}

After the experimental procedures were approved by Trakya University Animal Experimentations Ethics Committee, twenty male Sprague Dawley rats (twelve-weeks-old, weighing 250 to $350 \mathrm{~g}$ ) were obtained from the Experimental Animal Center of Trakya University. Animals were divided into two groups: control group $(n=8)$ and diabetes group $(n=12)$. All of the subjects were kept in convenient laboratory conditions (temperature $22 \pm 1$ ${ }^{0} \mathrm{C}, 12$ hours light/dark cycle), and maintained on a proper diet chow and water adlibitum.

\section{Chemicals}

Streptozotocin (STZ) was purchased from SigmaAldrich Corporation (St.Louis, MO, USA) dissolved in $0.1 \mathrm{M}$ citrate buffer, $\mathrm{pH} 4.2$.

\section{Experimental procedure}

The blood glucose levels were checked in all animals before streptozotocin/citrate buffer treatment. Blood glucose levels were no differences in control and diabetes groups $(88-111 \mathrm{mg} /$ $\mathrm{dl}$ and 90-112 mg/dl, respectively). While control group subjects were administered only $0.1 \mathrm{~mL}$ citrate buffer, diabetes group subjects were administered $40 \mathrm{mg} / \mathrm{kg}$ STZ during five consecutive days. Diabetes was confirmed by weekly measurement of blood glucose concentrations (above $250 \mathrm{mg} / \mathrm{dl}$ ) with glucometer. After the six weeks of induction of diabetes, all testes tissues were totally removed under the anesthesia with alfamine (Ketamidor, Alfasan, Holland) and rompun (Bayer, Turkey), body and testicular weights were measured and processed for examinations with light microscopy and immunohistochemistry.

Testes tissue samples were fixed with $10 \%$ formaldehyde, washed, dehydrated and finally embedded in paraffin (Merck, Darmstadt, Germany). $5 \mu \mathrm{m}$ sections were stained with hematoxylin $\& \operatorname{eosin}(\mathrm{H} \& \mathrm{E})$ to evaluate the histological structural features of testes. Using with same slides, mean seminiferous tubules diameters (MSTD) were determined with x200 magnification using ocular micrometer from the transversal sections of ten circular or circular-like random tubules ${ }^{4,11}$.

\section{Immunohistochemistry}

Formaldehyde-fixed, paraffin-embedded tissues were cut into $5 \mu \mathrm{m}$ sections. Slides were deparaffinized, rehydrated in a graded series of ethanol, and then boiled in citrate buffer $(10 \mathrm{mM}$; $\mathrm{pH}$ 6.0) for $20 \mathrm{~min}$ for antigen retrieval. Following washing in Trisbuffered saline (TBS), the sections were immersed in 3\% hydrogen peroxide for $5 \mathrm{~min}$. Slides were incubated with 5\% normal horse serum (VectorLaboratories, Burlingame, CA, USA) for PCNA and with 5\% normal goat serum (VectorLab.) for ERK and JNK in TBS for $30 \mathrm{~min}$ at room temperature in a humidified chamber. Excess serum was drained, and incubated in primary antibodies [monoclonal mouse, PCNA(F-2): sc-25280 (1:200 dilution in TBS; Santa Cruz BioTechnology, CA, USA); monoclonal rabbit p44/42 MAPK (ERK 1/2) and phospho-p44/42 MAPK (ERK 1/2) (Thr 202/ Tyr 204) (1:100 dilution in TBS; Cell Signaling Technology-CST, 
Beverly, MA, USA), polyclonal rabbit SAPK/JNK and phosphoSAPK/JNK (Thr183/Tyr185) (1:100 dilution in TBS; CST), overnight at $4^{\circ} \mathrm{C}$ in a humidified chamber. For negative controls, primary antibodies were replaced by their non-immune isotypes. Following washing three times for $5 \mathrm{~min}$ with TBS, sections were incubated with biotinylated horse anti-mouse and goat anti-rabbit antibodies (Vector Lab.) added at a 1:400 dilution for $30 \mathrm{~min}$ at room temperature. After washing three times with TBS, sections were incubated with a streptavidin-biotin-peroxidase kit (Vector Lab.) for an additional $30 \mathrm{~min}$ and the immunoreactivity was developed using DAB (3, 3-diaminobenzidine tetrahydrochloride dihydrate; Vector Lab.). Slides were slightly stained with hematoxylin and mounted with permount mounting medium (Fisher Chemicals, Spring Weld, NJ, USA).

In order to estimate the PCNA-index, PCNA-positive cells in seminiferous tubules 100 cell in three randomly chosen fields were counted in each slide. The cells with brown nuclear staining were considered positive. Both stained and nonstained germ cells were counted and the ratio of stained cells to the total number of germ cells, "PCNA index," was calculated for each seminiferous tubule. The average PCNA index in each case was obtained by dividing the sum of all PCNA indices by the number of seminiferous tubules in which the calculation was carried out ${ }^{11}$.

Total (t)/phospho (p)-ERK and t/p-JNK were semiquantitatively evaluated using a histological score (HSCORE) value, average score was used for statistical analysis, described previosuly ${ }^{12}$.

\section{Statistical analysis}

S0064 Minitab Release 13 programme (License Number: WCP1331.00197) of Trakya University, Faculty of Medicine, Center of Information Technologies has been used for statistical analysis and values were stated as median \pm standard deviation (SD). P values less than 0.005 were regarded as significant. Blood glucose levels (first, seventh and the last day), initial and final body weights, testis weights and the measurements of mean seminifer tubule diameters (MSTD) using with ocular micrometer in both groups were compared with Mann-Whitney U test.

The immunoreactivity data obtained by HSCORE method and PCNA index were analyzed by Mann-Whitney U test and $p$ values less than 0.005 were regarded as significant while the differences between both groups were compared with Wilcoxon test.The differences of the immunoreactivity data in terms of total and phospho were compared as two dependent variables.

\section{Results}

In our study, blood glucoselevels were measured two days after the STZ administration and values over $250 \mathrm{mg} /$ dl were accepted as diabetes. Blood glucose levels after STZ administration were significantly higher in the diabetes group than in controls ( $>250 \mathrm{mg} / \mathrm{dl} ; \mathrm{p}=0.001$; Table 1$)$.

At the beginning of the study, rats with higher body weights were placed in the diabetes group, although the body weights of the diabetes group were observed to decrease notably more than controls before they were sacrificed ( $\mathrm{p}=0.001$; Table 1 ).

Additionally, the weights of both testes were observed to decrease more in the diabetes group than the control group $(\mathrm{p}=0.001 ;$ Table 1$)$.

Mean seminiferous tubuli diameter (MSTD) values of the diabetes group were significantly lower than controls $(\mathrm{p}=0.001$; Table1).

TABLE 1 - Body and testis weights, MSTD values.

\begin{tabular}{lccc}
\hline & $\begin{array}{c}\text { Control } \\
\text { group } \\
\text { (n=8) }\end{array}$ & $\begin{array}{c}\text { Diabetic } \\
\text { group } \\
\text { (n=12) }\end{array}$ & P \\
\hline Blood glucose (mg/dl) & $110 \pm 7$ & $533 \pm 66$ & $=0.001^{*}$ \\
& $258 \pm 3$ & $303 \pm 27$ & \\
The first weight (gr) & $327 \pm 19$ & $179 \pm 17$ & $=0.001^{*}$ \\
& $1664 \pm 653$ & $691 \pm 374$ & $=0.001^{*}$ \\
The final weight (gr) & $1750 \pm 605$ & $697 \pm 333$ & $=0.001^{*}$ \\
$\begin{array}{l}\text { Right testis (gr) } \\
\text { Left testis (gr) }\end{array}$ & $308 \pm 9$ & $159 \pm 18$ & $=0.001^{*}$ \\
$\begin{array}{l}\text { Seminiferous tubules } \\
\text { diameter (MSTD- } \mu \mathrm{m})\end{array}$ & & & \\
\hline
\end{tabular}

*Diabetic group values compared with control group values, $\mathrm{p}<0.005$ was considered statisticaly significant.

\section{Immunohistochemical findings}

Testicular injury and spermatogenesis were evaluated histopathologically using H\&E slides (Figure 1A and 1C). When we compared findings for the two groups, the diabetes group's testis sections reflected all expected findings such as decrease in MSTD, vacuolization, and degeneration in spermatogenetic series cells. In diabetic rats, atrophy of the seminiferous tubules with varying degrees of spermatogenetic arrest was also observed (Figure 1C).

Counting spermatogenic cells for each group revealed that PCNA-positive cells were detected at a higher level in the control group (Figure 1B). The number of PCNA-positive cells was lower in the diabetic group (Figure 1D). The PCNA indexwas significantly decreased in the diabetes group compared with the control group ( $\mathrm{p}=0.004$, Table 2; Figure 1D and 1B, respectively). 


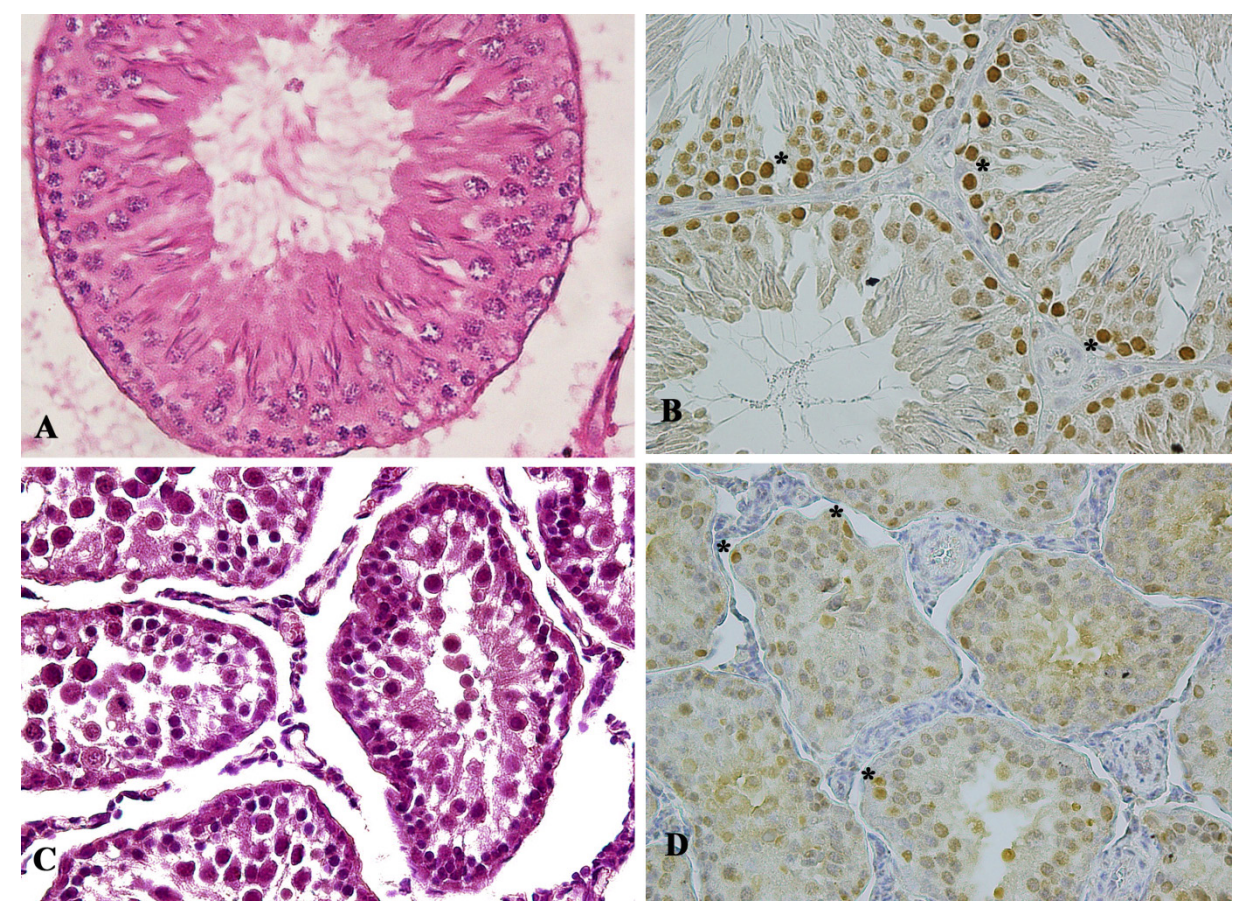

FIGURE 1 - Representative micrograph H\&E and PCNA staining in testis. Normal testicular architecture was seen in the control group (A) and C is reflected all changes in diabetic testes tissue. Positive cells of PCNA staining $(*)$ were decreased in diabetic group (D) compared with the control group (B). Original magnification: X400.

Regarding t-ERK immunoreactivity, the values were significantly lower in the diabetes group than in controls $(\mathrm{p}=0.004$, Table2; Figure 2B and 2A, respectively), while the decrease in p-ERK immunoreactivity was even more significant between the control and diabetes groups ( $\mathrm{p}<0.001$, Table 2 ; Figure $2 \mathrm{C}$ and $2 \mathrm{D}$, respectively).

While p-JNK immunoreactivity was higher in the diabetes group than in controls $(p=0.002$, Table 2 ; Figures $2 \mathrm{H}$ and $2 \mathrm{G}$, respectively), the $\mathrm{t}-\mathrm{JNK}$ immunoreactivity showed no change between the control and diabetes groups ( $\mathrm{p}=0.125$, Table2; Figures $2 \mathrm{E}$ and $2 \mathrm{~F}$, respectively).
TABLE 2 - PCNA index, t-ERK, p-ERK, t-JNK and p-JNK immunoreactivities.

\begin{tabular}{lccc}
\hline & $\begin{array}{c}\text { Control group } \\
(\mathrm{n}=8)\end{array}$ & $\begin{array}{c}\text { Diabetic group } \\
(\mathrm{n}=12)\end{array}$ & $\mathrm{P}$ \\
\hline PCNA-index & $77 \pm 10$ & $24 \pm 9$ & $=0.004^{*}$ \\
& $81(60-84)$ & $23(9-38)$ & \\
t-ERK & $175 \pm 16$ & $145 \pm 40$ & $=0.004^{*}$ \\
& $172(145-221)$ & $141(82-233)$ & \\
p-ERK & $180 \pm 53$ & $95 \pm 40$ & $<0.001^{*}$ \\
& $193(48-242)$ & $94(25-186)$ & \\
t-JNK & $99 \pm 20$ & $118 \pm 38$ & $=0.125$ \\
& $99(67-137)$ & $120(60-194)$ & \\
p-JNK & $194 \pm 32$ & $233 \pm 29$ & $=0.002^{*}$ \\
& $200(150-243)$ & $234(183-280)$ & \\
\hline
\end{tabular}

*Diabetic group values compared with the control group values, $\mathrm{p}<0.005$ was considered statisticaly significant.

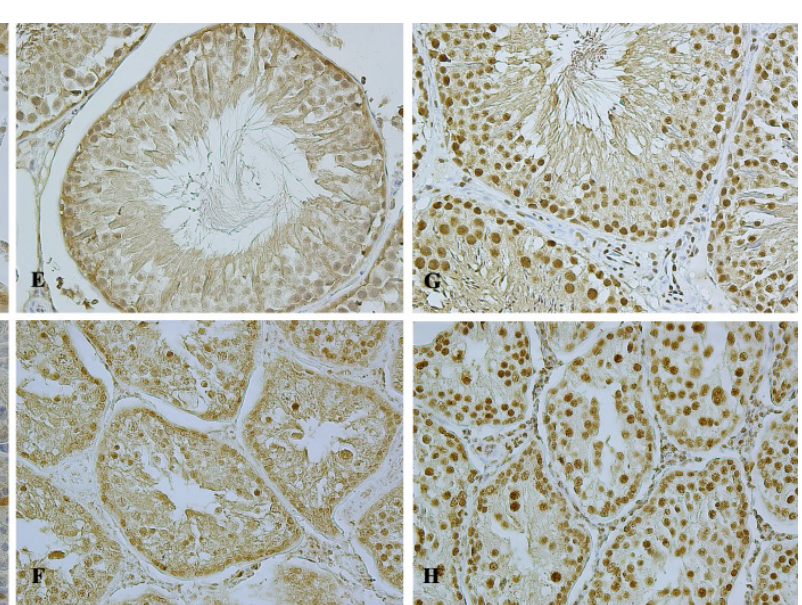

FIGURE 2 - Immunoreactivities in control and diabetic group for t/p-ERK and t/p-JNK. t-ERK and p-ERK immunoreactivity was both cytoplasmic and nuclear, with a higher intensity in control (A and $\mathbf{C}$, respectively) compared to diabetic group (B and $\mathbf{D}$, respectively). t-JNK immunreactivities were no differences in control (E) and diabetic group (F) but p-JNK immunoreactivities increased in diabetes group (H) than controls (G). Original magnification: x400. 


\section{Discussion}

Diabetes mellitus affects almost all organs and systems with either micro- or macrovascular complications such as retinopathy, nephropathy, neuropathy, etc. ${ }^{4}$. The pathophysiology of these complications are well known but the effects of diabetes on the male reproductive system remains obscure.

Our study's body and testis weightfindings have been supported by several authors like Altay et al. ${ }^{11}$. Aybek et al. ${ }^{4}$ suggested that the body weight of diabetic men increased and Cai et al. ${ }^{3}$ suggested that testis weight did not show any change at one month of follow-up but decreased after six months of diabetes.

Our findings about MSTD are similar to other studies ${ }^{11,14}$.

Histopathological changes have been observed in the germinal epithelium and the interstitial tissue due to diabetes in testis tissues of diabetic animals. In our study, damage to the spermatogenic cell line and detachment, germ cell loss in certain tubules, vacuolization in Sertoli cells, multinuclear giant cells, decreased sperm concentration in the tubular lumen, tubular atrophy, and invaginations from the walls of the tubules have been observed.

Cai et $a l^{3}$ have observed atrophy in the seminiferous tubules. Koh et al. ${ }^{7}$ have reported decrease in sperm counts, differentiation of Leydig cells, increase in the apoptosis of spermatogonium and spermatocytes, and abnormalities of the pituitary-testis axis. They suggested that decreases in testosterone levels, abnormal spermatogenesis, and germ cell apoptosis might be related to these changes.

In the diabetic group, the results of the PCNA index was in agreement with previous studies ${ }^{11}$. In our study, PCNA immunoreactivity was observed to be strong in the seminiferous tubules of the control group and especially spermatogonia, while moderate reactivity was observed in spermatocyte-I and spermatocyte-II. PCNA was nonreactive in Sertoli cells. In the diabetes group, moderate immunoreactivity was observed in a few germ cells and generally mild reactivity was observed. Altay et $a l{ }^{11}$ reported similar results in their study conducted with type I diabetic rats in 2003 .

Oxidative stress may develop with a decrease in insulin responsiveness, diabetes, and cardiovascular diseases. Reactive oxygen species (ROS) production may increase and insulin resistance may occur as a result of increased blood glucose and fatty acid levels ${ }^{15}$. There is a fine balance in the reproductive system between ROS production and the antioxidant protective system, and normally the level of ROS is quite low to regulate normal sperm functions ${ }^{4}$. The increase in ROS levels results in testes and sperm with oxidative damage and may lead to infertility. ROS levels have been observed to decrease in $40 \%$ of the infertile couples ${ }^{4,16}$. The relation between increased ROS levels and insulin resistance may be activated by some certain signal pathways. The DNA damage caused by ROS accelerates apoptosis and shows disadvantageous effects by decreasing sperm counts and results in infertility. The mRNA levels of stress-sensitive kinases such as JNK, p38, MAPK, and kappa B kinase inhibitor increase with the proinflammatory cytokines, including tumor necrosis factor- $\alpha$ (TNF- $\alpha$ ), interleukin- 6 (IL-6), and monocyte chemoattractant protein (MCP-1) by means of oxidative stress and ROS production. Systemic or local activation of TNF- $\alpha$, IL- 6 , and MCP-1 induces the production of ROS and a positive feedback mechanism takes place ${ }^{16}$.

The first member of the MAPK family that we have evaluated in our study is ERK, which is an important protein for tubule germinal epithelium proliferation. ERK is supposed to regulate the cellular proliferation by effecting the transcription of genes and phosphorylation of substrates. The significant decrease of p-ERK in diabetic subjects compared to t-ERK immunoreactivitymay be related to the decrease in germ cell proliferation. Moreover, the phosphorylation of ERK, which is produced by the interactions between spermatids and Sertoli cells, weakens communication between the adjacent proteins, thus facilitating the transport of mature sperm into the lumen ${ }^{17}$. The decreased number of sperm in the lumens of the diabetic testis samples may be related to the decreased levels of p-ERK caused by steroid hormones. The immunoreactivity evaluations of ERK, which is the member of the MAPK family responsible for cell proliferation, are consistent with our PCNA findings.

Zhang et al. ${ }^{18}$ suggested that IL-6 disrupts the bloodtestis barrier (BTB) integrity and changes the normal level and the regular localization of the BTB integral membrane proteins. It has also been shown that IL-6 has a role in inhibiting the degradation of BTB constitutive proteins and activating ERK-MAPK pathways. The results introduce a mechanistic understanding forthe function of IL-6 in regulation of BTB dynamics.

The JNK pathway is activated by cellular stress conditions such as oxidative stress and plays an important role in apoptosis, resistance to stress, and immune response. Phosphorylation of this pathway within the nucleus may activate stress response and apoptotic pathways. Acute oxidative stress leads to an increase of p-JNK within the nucleus while chronic oxidative stress leads to an increase of p-JNK within the cytoplasmic space. Oxidative stress and activated JNK may alter the expression of genes associated with diabetes ${ }^{15}$.

Koh suggested that western blotting analysis predicts a rise in $\mathrm{p}$-JNK expression in testes of diabetic rats ${ }^{2}$. In another study, Koh demonstrated hat there is a decrease in Bcl-2 and 
Bcl-XL levels and increase in Bax and Bad levelsin the testes of diabetic animals ${ }^{7}$.

Prause et al. ${ }^{19}$ showed that JNK functions as antiapoptotic in palmitate and high glucose-induced $\beta$-cell death. They also concluded that ROS and endoplasmic reticulum stress are associated with higher expression of proapoptotic Bcl-2 proteins in palmitate and high glucose-induced oxidative stress. To sum up, they propose that JNK1 prevents palmitate and high glucose-induced $\beta$-cell apoptosis, expression of puma, and formation of ROS.

Ranawat and Bansal suggested that a reduction of endogenous GSH as a result of selenite treatment in testicular cells is correlated with decreased $\mathrm{Bcl}-2$ expression, increased apoptosis, increased expression of p38 and JNK MAPK, and an increase in caspase- 3 expression ${ }^{16}$.

This is the first study that shows the level of JNK phosphorylation in diabetic male rats by immunohistochemistry. In our study, we observed that levels of t-JNK and f-JNK were noticeably higher in the diabetic group than in the control group and the immunoreactivity of t-JNK was also higher in the diabetic group,though it was statistically insignificant $(p=0.125$; Table 2 ), while f-JNK immunoreactivity was significantly increased in diabetic subjects $(\mathrm{p}=0.002$; Table 2$)$.

The JNK interference in cytokine-induced $\beta$-cell death was used to help understand the differential biological functions of JNK subtypes. It was found that JNK3 has antiapoptotic properties in cytokine-induced $\beta$-cell apoptosis. In addition, JNK 1 and JNK2 were shown to have proapoptotic functions in response to cytokines ${ }^{19}$.

Inflammatory issues play important roles in the pathogenesis of both type I and II diabetes. TNF- $\alpha$, IL- $1 \beta$, certain cytokines within the IL-6 family, IL-8 and some chemokines are active in the inflammatory process in both types of diabetes ${ }^{19}$. The plasma levels of IL- 6 are assumed to increase the risk of diabetes with regard to insulin resistance, regardless of body weight. Insulin resistancemay develop in healthy humans after the administration of high doses of IL-6. TNF- $\alpha$ inhibits insulin secretion induced by glucosis, which may damage the DNA of insulin and may induce $\beta$-cell apoptosis by means of Bcl-2. TNF- $\alpha$ has been demonstrated to be highly secreted in the adipose tissue and to play an important role in the development of insulin resistance. Adipocytokines such as leptin, TNF- $\alpha$, and IL- 6 have been identified as increasing the risk of diabetes ${ }^{20}$.

The increase of JNK levels in our diabetic subjects suggested that various cytokines such as TNF- $\alpha$, IL- $1 \beta$, and IL-6 and chemokines are released as a result of alterations in the expression of certain genes and proteins and therebyinduce apoptosis within the testis tissue. Furthermore, the decrease in the immunoreactivity of PCNA in the diabetes group indicated a lapse of proliferation in testis tissue while the increase in JNK may indicate the possible activation of the apoptotic pathway. The damage to the germ epithelium and the loss of cells may be attributed to these alterations.

Our findings may be utilized in the treatment of the patients with infertility due to diabetes; however, further and more advanced detailed studies are needed.

\section{Conclusion}

The decrease of androgen levels accompanying diabetes may contribute to the decrease of immunoreactivities of t-ERK and p-ERK. JNK may be activated by modification of the levels of certain genes and proteins by various cytokines and chemokines such as TNF- $\alpha$, IL- $1 \beta$, and IL- 6 that play roles in the regulation of diabetes, the process of oxidative stress, and apoptosis resulting in infertility.

\section{References}

1. Alves MG, Martins AD, Rato L, Moreira PI, Socorro S, Oliveira PF. Molecular mechanisms beyond glucose transport in diabetes-related male infertility. Biochim Biophys Acta. 2013 May;1832(5):626-35. doi: 10.1016/j.bbadis.2013.01.011.

2. Koh PO. Streptozotocin-induced diabetes increases apoptosis through JNK phosphorylation and Bax activation in rat testes. J Vet Med Sci. 2007 Sep;69(9):969-71. PMID: 17917385.

3. Cai L, Chen S, Evans T, Deng DX, Mukherjee K, Chakrabarti S. Apoptotic germ-cell death and testicular damage in experimental diabetes: prevention by endothelin antagonism. Urol Res. 2000 Oct;28(5):342-7. PMID:11127715.

4. Aybek H, Aybek Z, Rota S, Sen N, Akbulut M. The effects of diabetes mellitus, age, and vitamin $\mathrm{E}$ on testicular oxidative stress. Fertil Steril. 2008 Sep;90(3):755-60. PMID: 17482598.

5. Baccetti B, La Marca A, Piomboni P, Capitani S, Bruni E, Petraglia $\mathrm{F}$, De Leo V. Insulin-dependent diabetes in men is associated with hypothalamo-pituitary derangement and with impairment in semen quality. Hum Reprod. 2002 Oct;17(10):2673-7. PMID: 12351547.

6. Kyathanahalli C, Bangalore S, Hanumanthappa K, Muralidhara. Experimental diabetes-induced testicular damage in prepubertal rats. J Diabetes. 2014 Jan;6(1):48-59. doi: 10.1111/1753-0407.12068.

7. Koh PO. Streptozotocin-induced diabetes increases the interaction of $\mathrm{Bad} / \mathrm{Bcl}-\mathrm{XL}$ and decreases the binding of $\mathrm{pBad} / 14-3-3$ in rat testis. Life Sci. 2007 Sep 8;81(13):1079-84. PMID: 17870134.

8. Zhao Y, Tan Y, Dai J, Li B, Guo L, Cui J, Wang G, Shi X, Zhang X, Mellen N, Li W, Cai L. Exacerbation of diabetes-induced testicular apoptosis by zinc deficiency is most likely associated with oxidative stress, p38 MAPK activation, and p53 activation in mice. Toxicol Lett. 2011 Jan 15;200(1-2):100-6. doi: 10.1016/j.toxlet.2010.11.001.

9. Jiang X, Zhang C, Xin Y, Huang Z, Tan Y, Huang Y, Wang Y, Feng W, Li X, Li W, Qu Y, Cai L. Protective effect of FGF21 on type 1 diabetes-induced testicular apoptotic cell death probably via both mitochondrial- and endoplasmic reticulum stress-dependent pathways in the mouse model. Toxicol Lett. 2013 May 10;219(1):6576. doi: 10.1016/j.toxlet.2013.

10. Roy S, Metya SK, Rahaman N, Sannigrahi S, Ahmed F. Ferulic acid in the treatment of post-diabetes testicular damage: relevance to the down regulation of apoptosis correlates with antioxidant status via 
modulation of TGF- $\beta 1$, IL-1 $\beta$ and Akt signalling. Cell Biochem Funct. 2014 Jan;32(1):115-24. doi: 10.1002/cbf.2983.

11. Altay B, Cetinkalp S, Doganavşargil B, Hekimgil M, Semerci B. Streptozotocin-induced diabetic effects on spermatogenesis with proliferative cell nuclear antigen immunostaining of adult rat testis. Fertil Steril. 2003 Sep;80 Suppl 2:828-31. PMID: 14505760.

12. Kizilay G, Cakmak H, Yen CF, Atabekoglu C, Arici A, Kayisli UA. Expression and regulation of c-Jun N-terminal kinase (JNK) in endometrial cells in vivo and in vitro. Histochem Cell Biol. 2008 Oct;130(4):761-71. doi: 10.1007/s00418-008-0421-z.

13. Wada T, Penninger JM. Mitogen-activated protein kinases in apoptosis regulation. Oncogene. 2004 Apr 12;23(16):2838-49. PMID: 15077147.

14. Trindade AA, Simões AC, Silva RJ, Macedo CS, Spadella CT. Long term evaluation of morphometric and ultrastructural changes of testes of alloxan-induced diabetic rats. Acta Cir Bras. 2013 Apr;28(4):256-65. PMID: 23568233.

15. Berdichevsky A, Guarente L, Bose A. Acute oxidative stress can reverse insulin resistance by inactivation of cytoplasmic JNK. J Biol Chem. 2010 Jul 9;285(28):21581-9. doi: 10.1074/jbc.M109.093633.

16. Ranawat P, Bansal MP. Decreased glutathione levels potentiate the apoptotic efficacy of selenium: possible involvement of p38 and JNK MAPKs--in vitro studies. Mol Cell Biochem. 2008 Feb;309(12):21-32. PMID: 17989939.

17. Cheng J, Watkins SC, Walker WH. Testosterone activates mitogen-activated protein kinase via Src kinase and the epidermal growth factor receptor in sertoli cells. Endocrinology. 2007 May;148(5):2066-74. PMID: 17272394.

18. Zhang H, Yin Y, Wang G, Liu Z, Liu L, Sun F. Interleukin-6 disrupts blood-testis barrier through inhibiting protein degradation or activating phosphorylated ERK in Sertoli cells. Sci Rep. 2014 Mar 3;4:4260. doi: $10.1038 /$ srep04260.
19. Prause M, Christensen DP, Billestrup N, Mandrup-Poulsen T. JNK1 protects against glucolipotoxicity-mediated beta-cell apoptosis. PLoS One. 2014 Jan 24;9(1):e87067. doi: 10.1371/journal. pone. 0087067

20. Hajer GR, van Haeften TW, Visseren FL. Adipose tissue dysfunction in obesity, diabetes, and vascular diseases. Eur Heart J. 2008 Dec;29(24):2959-71. doi: 10.1093/eurheartj/ehn387.

\section{Correspondence:}

Associate Professor Gulnur Kizilay Trakya University, Faculty of Medicine, Department of Histology and Embryology 22030, Edirne/Turkey

Phone: +(90) 284 2357642-1402

Fax Number: +(90) 2842355935

gulnurkizilay@trakya.edu.tr

gkzlay@yahoo.com

Received: May 19, 2014

Review: July 18, 2014

Accepted: Aug 22, 2014

Conflict of interest: none

Financial source: Trakya University Research Center (Project $\mathrm{n}^{\mathrm{o}}$ : 2008/116)

${ }^{1}$ Research performed at Department of Histology and Embryology, Faculty of Medicine, Trakya University, Edirne, Turkey. 